\title{
Antibiotics Resistance, Sensitivity Pattern and Development of Antibiogram to Support Empirical Prescription in Health Facilities in South Senatorial District of Kwara State, Nigeria
}

\author{
Gbenga Solomon Joseph ${ }^{1 *}$, Adekunle Ganiyu Salaudeen ${ }^{2}$ \\ ${ }^{1}$ School of Public Health, Texila American University, Georgetown, Guyana, South America \\ ${ }^{2}$ Department of Epidemiology and Community Health, University of Ilorin Teaching \\ Hospital, Ilorin, Nigeria
}

\begin{abstract}
A major concern in public health is Antibiotic resistance among pathogenic microorganisms. There are several organisms developing resistance to commonly used antibiotics in different locations and time. This study assessed patterns of antibiotic resistance in line with the WHO classification of antibiotics. A cross-sectional study was carried out from September 2020 to January 2021 in 4 randomly selected hospitals. Of all 442 patients recruited for this study, twenty-seven percent (122) were randomly selected for Antimicrobial Susceptibility Testing using the modified Kirby-Bauer disk diffusion method, and SPSS version 23 was used for analysis. The most predominant isolate was Staphylococcus aureus. Resistance by Staphylococcus aureus was noted against Amoxicillin, Trimethoprim-Sulfamethoxazole, Amoxicillin clavulanic acid, Gentamicin, and Cefuroxime. Resistance by E. coli against Chloramphenicol 80\%, cefuroxime 100\%, Trimethoprim-Sulfamethoxazole $83.3 \%$, Resistance by Klebsiella spp; against Ampicillin Second and third-generation cephalosporin and Gentamicin 100\%. Resistance by Streptococcus spp; Ampicillin 100\% and Ampicillin cloxacillin 100\%, cefuroxime 100\%, and Ciprofloxacin 100\%. Ciprofloxacin and levofloxacin were active against Salmonella species, Pseudomonas species, klebsiella, and Escherichia coli. Azithromycin, imipenem, and meropenem with high susceptibility to local bacterial isolates sample tested. The access group antibiotics showed a low susceptibility rate to local bacteria isolate with increase resistance to the watch group. Antibiogram was developed to guide empirical treatment type that is predominant in the district. Most bacteria isolated from health facilities in Kwara South were resistant to the access group. The resistance to watch a group of WHO antibiotics classification is building very fast.
\end{abstract}

Keywords: Antibiotic Resistance, Empirical Treatment, Susceptible.

\section{Introduction}

The most commonly used and abused antimicrobial agent in the management of bacterial infection globally is antibiotics agents [1]. A major concern in public health is Antibiotic resistance among pathogenic microorganisms. The increasing prevalence of antimicrobial resistance is putting pressure on the healthcare system, and this has become a threat to public health. It has increased resistance to first-line antibiotics and increased resistance to second-line antibiotics in the community, with consequences increasing deaths and costly treatment [2]. There are several organisms developing resistance to commonly used antibiotics in different locations and times. Antibiotic resistance (ABR) impedes the effective prevention and treatment of a continuous increasing range of bacterial infections [1].

Resistance in Klebsiella pneumoniae resulted in life-threatening infections to reserve a group of antibiotics. K. pneumoniae is the main cause 
of hospital-acquired infections such as pneumonia, bloodstream infections, and infections in newborns and intensive-care unit patients [3]. In a study conducted in 2019 in Brazil intensive care unit of the hospital, the author observed Klebsiella pneumoniae developed resistance to beta-lactam antibiotics: ampicillin/sulbactam, piperacillin/tazobactam, cefuroxime, cefoxitin, ceftazidime, ceftriaxone, cefepime, ertapenem, imipenem and meropenem. This revealed that Klebsiella pneumoniae isolates demonstrate different degrees of resistance to other antibiotics like gentamicin (80\%), Ciprofloxacin (64\%), TGC (52\%), CST (36\%), and amikacin (4\%) [4].

In a study conducted in Ibadan, Nigeria, the author observed, resistance to a common antibiotic is increasing; the author documented E. coli isolates showed resistance to ciprofloxacin $(92.86 \%), \quad$ cotrimozazole $(92.86 \%)$, and ceftriazone $(78.58 \%)$. He also observed a lower susceptibility to oflaxacin $(28.57 \%)$ in the study [5]. Similarly, in another study in southwestern Nigeria, among food sellers on the campus of the University of AdoEkiti, the antibiotic resistance pattern of the isolates showed six out of the eight antibiotics tested was resistance to commonly bacteria isolate [6]. This shows a higher resistance by microorganisms in Nigeria. In a study conducted in Kano and Borno University Teaching Hospitals among the patients on admission, it was observed that Staphylococcus aureus develops resistance very quickly and successfully to different antimicrobials over a period. The highest frequency of S. aureus occurred with susceptibility to antimicrobial agent Levofloxacin (100\%) followed by Ciprofloxacin (78.9\%), while the least was Penicillin (7.1\%) [7].

The situation report presented by Nigeria Centre for Disease Control revealed a trend of the wide spread of resistance strain to watch a group of antibiotics according to World Health Organization categorization [7]. In a study in Port Harcourt, Rivers State, Nigeria, it was observed that the Upper respiratory tract infection $(83.7 \%)$ and diarrhoea $(55.9 \%)$ were significantly associated with empirical antibiotic prescription $(\mathrm{P}=0.05$ and 0.002 [8]. Empirical prescription contributes greatly to antibiotic resistance.

Several factors have been found to contribute to antimicrobial resistance epidermic, especially in poor resource settings: the triple threat of unfettered access, minimal product regulation and failure to ensure appropriate guideline on antibiotic prescription is followed, and Lack of clinical diagnostic tools to support antibiotic resistance control amongst others [9]. Antibiotics resistance epidermic in Nigeria is facilitated by inappropriate medicine use, poor infection prevention, and control, Lack of government commitment to control of antimicrobial resistance and poor AMR surveillance system in place, and Lack of antimicrobial stewardship in both private and public sectors. In Nigeria, antimicrobial surveillance is weak. Antibiotics are prescribed unnecessarily and empirically for complaints where no antibiotic is needed or where culture and sensitivity results could safely be awaited (AMR use and situation analysis 2017). The empirical prescription is not guided by an antibiotic's stewardship instrument like an antibiogram that tracked and monitored resistance and susceptible strains in a given time and location.

Antimicrobial use and situation analysis in Nigeria identify setting up a national surveillance system for AMR, strengthen institutional capacities for early AMR detection and trends monitoring in the country, build laboratory capacity to produce high-quality microbiological data across all sectors, and implement a research agenda for AMR burden as a solution to the current antibacterial resistance burden in Nigeria. The strategic solution was to promote research in Government and private hospital and strengthen institutional capacities for early AMR detection and trends monitoring in the country. The monitoring of antibiotic use 
in Nigeria is still weak in many institutions in Nigeria. Though a lot has been done to create awareness to promote antibiotic's rational use, The Lack of research to quantify the resistance burden at the institution and subnational level and data to support antibiotic monitoring is still a challenge in Nigeria.

To optimize the use of antimicrobials and to pave the way for the implementation of antibiotic stewardship in the hospital [10], the World Health Organization (WHO) in 2017 updated the Essential Medicine List (EML) and categorized antibiotics into three groupsAccess, Watch and Reserve (AWaRe). This strategy aimed to ensure that antibiotics are available when needed and that the appropriate antibiotics are prescribed for the right infections. It would improve treatment outcomes, reduce the development of drug-resistant bacteria, and preserve the effectiveness of "last resort" antibiotics that are needed when all others failed [10].

In the face of the growing burden of antibiotics resistance, there is a dearth in research and data on this subject in Kwara State and Nigeria, a resource-limited setting [7]. A study to determine antibiotics resistance burden and sensitivity pattern to develop an antibiogram that will improve empirical treatment outcome and inform the development of appropriate policies for establishing antibiotics stewardship is required in this setting.

Therefore, this study was carried out to investigate the prevalence of antibiotics resistance and susceptibility patterns of bacteria isolated from samples collected from Government and Private hospitals in Kwara south senatorial district to develop an antibiogram that can be updated continuously to guide empirical treatment and rational use of antibiotics.

Estimating antibiotics resistance and sensitivity using (AWaRe) WHO classification to develop an antibiogram for the hospital is a new concept in Kwara south senatorial district. Antibiogram is a tool that guides empirical treatment type and antibiotic formulary for the hospital in the Kwara south with the goal of improving clinical outcomes.

\section{Material and Methods}

A cross-sectional study of antibiotics resistance and sensitivity pattern to the bacterial pathogen in Kwara south senatorial district was carried out on 442 patients between September 2020 and January 2021. Approval from the State Ministry of Health Research and Ethical Committee was obtained prior to the commencement of the study. A multistage sampling technique was used. Out of three senatorial districts in Kwara State, Kwara South senatorial district was selected using simple random sampling by balloting. The list of the Government secondary health facility and Private secondary health facility located in Kwara senatorial was obtained from the State Ministry of Health and used as the sampling frame. Two hospitals from the Government and private hospitals were selected from the Government and private hospitals list using simple random sampling. Using eligibility criteria, hospital patient registers from private and secondary health facilities selected were screened to have a list of eligible respondents for the sampling frame for this study. Systematic Sampling techniques were used to select respondents. The first respondent was selected by simple random sampling, while the subsequent respondents was selected using sampling interval.

A proportionate sampling method was used to determine the number of respondents from private and Government health facilities using the patient load in the four selected health hospitals. Of all 442 patients recruited for this study, twenty-seven percent (122) were randomly selected for Antimicrobial Susceptibility Testing (AST) WHO questionnaire on antibiotic resistance prevalence survey was modified and adopted to collect data regarding the socio-demographic data of the respondents. 
All bacteria were identified by standard microbiologic methods. The antibiotic susceptibility testing was done by using the modified Kirby-Bauer disk diffusion method. The antibiotic disks which were used were ampicillin, ampicillin/ sulbactam, ceftazidime, cefotaxime, cefuroxime, cefaperazone/ sulbactam, nitrofurantoin, co-trimoxazole, gentamicin, amikacin, norfloxacin, ciprofloxacin, piperacillin, piperacillin/ tazobactum, imipenem, and meropenem.

The zone size around each antimicrobial disk was interpreted as sensitive, intermediate, or resistant according to the Clinical and Laboratory Standards Institute (CLSI) criteria. Organisms sensitive and resistant to the drug were documented. Data regarding culture and sensitivity of the organisms isolated from different sources from urine, blood, wound swab/pus, stool, sputum, and tracheal aspirations were collected from both out-patients (OP) and in-patients (IP). All data were tabulated and analyzed. Descriptive and inferential statistics were used for analysis, and the results were expressed.

\section{Description of the study Area}

This study was conducted in Kwara South Senatorial District (KSSD) of Kwara State. The Language of the people of Kwara south senatorial district is mainly Yoruba with three sub-ethnic nationalities, namely, Ekiti, Ibollo, and Igbomina. There are other ethnic groups like Bassa, Fulani, Hausa, Igbo, Nupe, and Tiv living in the area. Kwara south senatorial district has guinea savannah vegetation. This senatorial district geographically shared boundaries with Kwara central senatorial district to the west, Kwara north senatorial district to the North, Kogi State to the east, and with the duo of Ekiti and the Osun States to the South.

The levels of care in the public sector are primary, secondary. The primary level is financed and managed by the local government, and the secondary is by the state. Primary health care (PHC) facilities exist at all the Local
Government Areas (LGAs); they form the entry point of the community into the health care system. PHC facilities provide preventive, curative, promotive, and pre-referral care to the population. PHC facilities are typically staffed by nurses, community health workers, community health extension workers (CHEWs), junior CHEWs, and environmental health officers. They are headed technically and administratively by the PHC coordinator, normally the Medical Officer of Health (MOH) where one exists who is assisted by program officers and unit heads.

The secondary health facilities referred to as general hospitals provide medical and laboratory services, as well as specialized health services such as surgery, paediatrics, obstetrics, and gynaecology to patients referred from the PHC level. Medical officers, nurses, midwives, pharmacists, laboratory specialists, and community health officers are the typical staff at secondary health facilities. They are headed by Medical Directors.

The study was conducted in four secondary health facilities selected by simple random sampling: General Hospital Omu-Aran and General Hospital, Offa (representing public health facilities) and Private Hospital are ESquare Hospital, Oro and Olalomi Hospital, Erin-ile. These health facilities are located in four LGAs of the seven LGAs in the senatorial district.

\section{Results}

Over a quarter $(27.6 \%)$ of participants had sample collection for culture, and $98.4 \%$ of participants that had sample collection had their results reported. Almost two-thirds (62.3\%) of the sample taken had growth of different types of microorganisms. Over eight different types of specimens were taken, with urine having the highest proportion (42.6\%). This was distantly followed by a sputum/respiratory tract sample that accounted for $13.1 \%$. The blood sample was the least with a $0.8 \%$ proportion (Table 1 ). 
Table 1. Specimen taken for Culture and Results before Antibiotics Prescription

\begin{tabular}{|l|l|l|}
\hline Variable & Frequency & Percent \\
\hline Culture sample taken $(\mathbf{n}=442)$ & \multicolumn{2}{|l|}{} \\
\hline Yes & 122 & 27.6 \\
\hline No & 320 & 72.4 \\
\hline Specimen type (n= 122) & 1 & 0.8 \\
\hline Blood & \multicolumn{2}{|l|}{} \\
\hline Urine & 52 & 42.6 \\
\hline Sputum/Respiratory sample & 16 & 13.1 \\
\hline Wound & 12 & 9.8 \\
\hline High Vaginal Swab & 14 & 11.5 \\
\hline Pus & 13 & 10.7 \\
\hline Stool & 4 & 3.3 \\
\hline Semen & 3 & 2.5 \\
\hline Others & 7 & 5.7 \\
\hline Culture results $(\mathbf{n}=\mathbf{1 2 2})$ & & 62.3 \\
\hline Growth & 76 & 36.1 \\
\hline No growth & 44 & 1.6 \\
\hline Not available & 2 & \\
\hline
\end{tabular}

The highest number (24) of growth reported The least reported were pseudomonas specie and was for Staphylococcus aureus 24 (31.58), salmonella specie $1(1.32 \%)$. closely followed by the coliform organism (22).

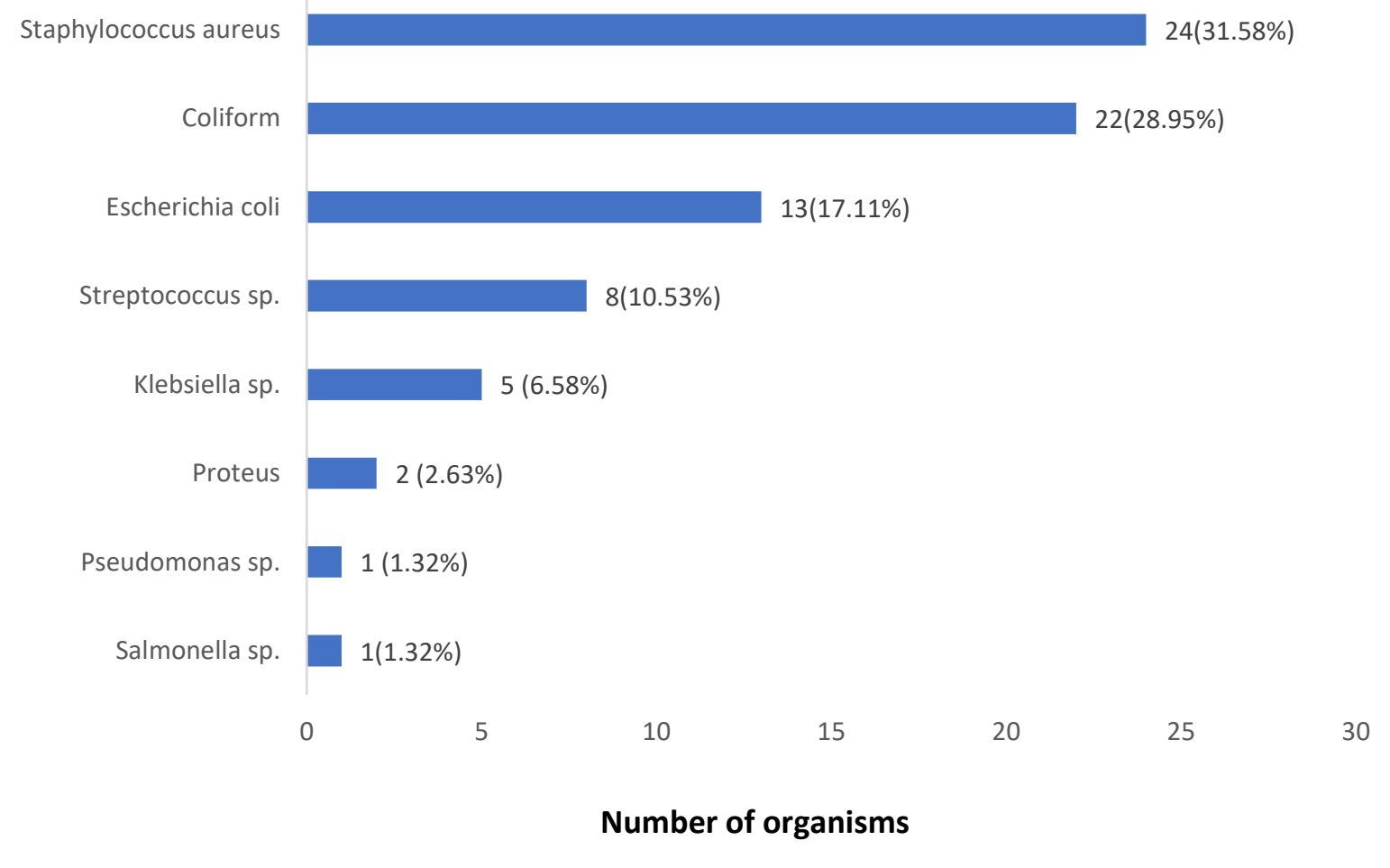

Figure 1. Distribution of Bacterial Organisms Grown from Samples 
Table 2. Different Bacterial Organisms Grown in the Culture Test of the Specimen

\begin{tabular}{|c|c|c|c|c|c|c|c|c|c|c|c|c|c|c|c|c|}
\hline & \multicolumn{2}{|c|}{$\begin{array}{l}\text { Streptococcus } \\
\text { sp }\end{array}$} & \multicolumn{2}{|c|}{$\begin{array}{l}\text { Staph. } \\
\text { aureus }\end{array}$} & \multicolumn{2}{|c|}{$\begin{array}{l}\text { Salmonella } \\
\text { sp }\end{array}$} & \multicolumn{2}{|c|}{$\begin{array}{l}\text { Pseudomonas } \\
\text { sp }\end{array}$} & \multicolumn{2}{|c|}{ Proteus } & \multicolumn{3}{|c|}{ Coliform } & \multicolumn{2}{|c|}{$\begin{array}{l}\text { Klebsiella } \\
\text { sp }\end{array}$} & \multirow[t]{2}{*}{ Escherichia } \\
\hline Antibiotics tested & $\mathbf{R}$ & & $\mathbf{R}$ & & $\mathbf{R}$ & & $\mathbf{R}$ & & $\mathbf{R}$ & & $\mathbf{R}$ & & & $\mathbf{R}$ & & \\
\hline Ampicillin Cloxacillin & $3 / 3$ & 100 & $2 / 2$ & 100 & - & - & - & - & - & - & $6 / 7$ & 85.7 & $1 / 1$ & 100 & $2 / 2$ & 100 \\
\hline Ampicillin & - & - & - & - & - & - & - & - & - & - & $1 / 1$ & 100 & & & $1 / 1$ & 100 \\
\hline Amoxicillin & $5 / 5$ & 100 & $10 / 12$ & 83.3 & - & - & - & - & $2 / 2$ & 100 & $1 / 3$ & 33.3 & $4 / 4$ & 100 & $4 / 9$ & 44.4 \\
\hline Amoxicillin Clavulanic acid & $1 / 3$ & 33.3 & $9 / 14$ & 64.3 & - & - & - & - & $2 / 2$ & 100 & $11 / 13$ & 84.6 & $3 / 3$ & 100 & $5 / 9$ & 55.6 \\
\hline Azithromycin & - & - & & & - & - & - & - & - & - & - & - & - & - & - & - \\
\hline Ceftazidime & - & - & $1 / 1$ & 100 & - & - & - & - & - & - & - & - & $1 / 1$ & 100 & - & - \\
\hline Cefixime & - & - & - & - & - & - & - & - & - & - & $1 / 2$ & 50.0 & - & - & - & - \\
\hline Cefazolin & - & - & - & - & - & - & - & - & - & - & & & $3 / 3$ & 100 & - & - \\
\hline Chloramphenicol & $2 / 5$ & 40.0 & $3 / 5$ & 60.0 & $1 / 1$ & 100 & $1 / 1$ & 100 & - & - & $4 / 4$ & 100 & & & $4 / 5$ & 80.0 \\
\hline Ciprofloxacin & $3 / 3$ & 100 & $1 / 14$ & 7.1 & - & - & - & - & - & - & $2 / 11$ & 18.2 & $1 / 5$ & 20.0 & $1 / 7$ & 14.3 \\
\hline Clarithromycin & - & - & - & 0.0 & - & - & - & - & - & - & - & - & - & - & - & - \\
\hline Cefuroxime & $3 / 3$ & 100 & $2 / 4$ & 50.0 & - & - & - & - & - & - & - & - & $1 / 1$ & 100 & $5 / 5$ & 100 \\
\hline Ceftriaxone & $2 / 4$ & 50.0 & $3 / 8$ & 37.5 & - & - & - & - & - & - & - & - & - & - & - & - \\
\hline Erythromycin & $1 / 3$ & 33.3 & $7 / 11$ & 63.6 & - & - & - & - & - & - & $3 / 6$ & 50.0 & - & - & - & - \\
\hline Nitrofurantoin & - & - & $3 / 4$ & 75.0 & - & - & - & - & - & - & $4 / 8$ & 50.0 & - & - & - & - \\
\hline Gentamicin & $3 / 3$ & 50.0 & $11 / 17$ & 64.7 & - & & $1 / 1$ & 100 & $1 / 2$ & 50.0 & $10 / 17$ & 58.8 & $2 / 2$ & 100 & $3 / 8$ & 37.5 \\
\hline Imipinem & - & - & - & - & - & - & - & - & - & - & - & & - & - & - & - \\
\hline Levofloxacin & - & - & - & - & - & - & - & - & - & - & $1 / 8$ & 12.5 & - & - & - & - \\
\hline Meropinem & - & - & - & - & - & - & - & - & - & - & - & - & - & - & - & - \\
\hline Metronidazole & - & - & - & - & - & - & - & - & - & - & - & - & - & - & - & - \\
\hline Ofloxacin & $2 / 6$ & 33.3 & $9 / 18$ & 50.0 & - & & $1 / 1$ & 100 & $1 / 2$ & 50.0 & $3 / 16$ & 18.8 & $1 / 4$ & 25.0 & $1 / 7$ & 14.3 \\
\hline Streptomycin & - & - & $4 / 12$ & 33.3 & $1 / 1$ & 100 & - & - & - & - & $4 / 4$ & 100 & $1 / 3$ & 33.3 & $2 / 6$ & 33.3 \\
\hline Tetracycline & - & - & - & - & - & - & - & - & - & - & $1 / 1$ & 100 & $1 / 1$ & 100 & - & - \\
\hline Trimethoprim - Sulfamethoxazole & $3 / 5$ & 60.0 & $12 / 13$ & 92.3 & $1 / 1$ & 100 & $1 / 1$ & 100 & - & - & $5 / 7$ & 71.4 & $3 / 3$ & 100 & $5 / 6$ & 83.3 \\
\hline
\end{tabular}


Table 3. Antibiogram for the Health Facilities

\begin{tabular}{|c|c|c|c|c|c|c|c|c|c|c|c|c|c|c|c|c|}
\hline \multirow[b]{2}{*}{ Antibiotics tested } & \multicolumn{2}{|c|}{$\begin{array}{l}\text { Streptococcus } \\
\text { sp }\end{array}$} & \multicolumn{2}{|c|}{$\begin{array}{l}\text { Staph. } \\
\text { aureus }\end{array}$} & \multicolumn{2}{|c|}{$\begin{array}{l}\text { Salmonella } \\
\text { sp }\end{array}$} & \multicolumn{2}{|c|}{$\begin{array}{l}\text { Pseudomonas } \\
\text { sp }\end{array}$} & \multicolumn{2}{|c|}{ Proteus } & \multicolumn{2}{|c|}{ Coliform } & \multicolumn{2}{|c|}{ Klebsiella sp } & \multicolumn{2}{|c|}{ Escherichia } \\
\hline & $\mathbf{R}$ & $\mathbf{S}$ & $\mathbf{R}$ & $\mathbf{S}$ & $\mathbf{R}$ & $\mathbf{S}$ & $\mathbf{R}$ & $\mathbf{S}$ & $\mathbf{R}$ & $\mathbf{S}$ & $\mathbf{R}$ & $\mathbf{S}$ & $\mathbf{R}$ & $\mathbf{S}$ & $\mathbf{R}$ & $\mathbf{S}$ \\
\hline Ampicillin Cloxacillin & 100 & 0.0 & 100 & 0.0 & - & - & - & - & - & - & 85.7 & 14.3 & 100 & 0.0 & 100 & 0.0 \\
\hline Ampicillin & - & - & - & - & - & - & - & - & - & - & 100 & - & - & - & 100 & 0.0 \\
\hline Amoxicillin & 100 & 0.0 & 83.3 & 16.2 & 0.0 & 100 & - & - & 100 & 0.0 & 33.3 & 66.7 & 100 & 0.0 & 44.4 & 55.6 \\
\hline Amoxicillin Clavulanic acid & 33.3 & 66.7 & 64.3 & 35.7 & - & - & - & - & 100 & 0.0 & 84.6 & 15.4 & 100 & 0.0 & 55.6 & 44.4 \\
\hline Azithromycin & - & - & - & - & - & - & - & - & - & - & 0.0 & 100 & - & - & - & - \\
\hline Ceftazidime & - & - & 100 & 0.0 & - & - & - & - & - & - & - & - & 100 & 0.0 & - & - \\
\hline Cefixime & - & - & - & - & - & - & - & - & - & - & 50.0 & 50.0 & - & - & - & - \\
\hline Cefazolin & - & - & - & - & - & - & - & - & - & - & - & - & 100 & 0.0 & - & - \\
\hline Chloramphenicol & 40.0 & 60.0 & 60.0 & 40.0 & 100 & 0.0 & 100 & - & - & - & 100 & 0.0 & 0.0 & 100 & 80.0 & 20.0 \\
\hline Ciprofloxacin & 100 & 0.0 & 7.1 & 92.9 & - & - & - & 100 & 0.0 & 100 & 18.2 & 81.8 & 20.0 & 80.0 & 14.3 & 85.7 \\
\hline Clarithromycin & - & - & 0.0 & 100 & - & - & - & - & - & - & - & - & - & - & - & - \\
\hline Cefuroxime & 100 & 0.0 & 50.0 & 50.0 & - & - & - & - & - & - & 0.0 & 100 & 100 & 0.0 & 100 & 0.0 \\
\hline Ceftriaxone & 50.0 & 50.0 & 37.5 & 62.5 & - & - & - & - & - & - & - & - & 0.0 & 100 & - & - \\
\hline Erythromycin & 33.3 & 66.7 & 63.6 & 36.4 & - & - & - & - & - & - & 50.0 & 50.0 & - & - & - & - \\
\hline Nitrofurantoin & - & 100 & 75.0 & 25.0 & - & - & - & - & - & - & 50.0 & 50.0 & - & - & 0.0 & 100 \\
\hline Gentamicin & 50.0 & 50.0 & 64.7 & 35.3 & - & - & 100 & 0.0 & 50.0 & 50.0 & 58.8 & 41.2 & 100.0 & 0.0 & 37.5 & 62.5 \\
\hline Imipinem & - & - & - & - & - & - & - & - & - & - & - & - & - & - & 0.0 & 100 \\
\hline Levofloxacin & 0.0 & 100 & 0.0 & 100 & - & - & - & - & - & - & 12.5 & 87.5 & - & - & - & - \\
\hline Meropinem & - & - & - & - & - & - & - & - & - & - & - & - & - & - & 0.0 & 100 \\
\hline Metronidazole & 0.0 & 100 & - & - & - & - & - & - & - & - & - & - & - & - & 0.0 & 100 \\
\hline Ofloxacin & 33.3 & 66.7 & 50.0 & 50.0 & 0.0 & 100 & 100 & 0.0 & 50.0 & 50.0 & 18.8 & 81.3 & 25.0 & 75.0 & 14.3 & 85.7 \\
\hline Streptomycin & 0.0 & 100 & 33.3 & 66.7 & 100 & 0.0 & 0.0 & 100 & - & - & 100 & 0.0 & 33.3 & 66.7 & 33.3 & 66.7 \\
\hline Tetracyclin & - & - & - & - & - & - & - & - & - & - & 100 & 0.0 & 100 & 0.0 & - & - \\
\hline Trimethoprim - Sulfamethoxazole & 60.0 & 40.0 & 92.3 & 7.7 & 100 & 0.0 & 100 & 0.0 & - & - & 71.4 & 28.6 & 100 & 0.0 & 83.3 & 16.7 \\
\hline
\end{tabular}


The highest resistance by Staphylococcus aureus was noted against Amoxicillin 100\%, Ampicillin cloxacillin 100\%, Ciprofloxacin $100 \%$, Cefuroxime $100 \%$, and TrimethoprimSulfamethoxazole $60 \%$. Resistance by E. coli was noted against to Ampicillin 100\% and Ampicillin cloxacillin 100\%, Chloramphenicol $80 \%$, cefuroxime $100 \%$, TrimethoprimSulfamethoxazole $\quad 83.3 \% \quad$ Amoxicillin Clavulanic acid 55.6\%. Resistance by Klebsiella $s p$ was observed against Ampicillin 100\% and Ampicillin cloxacillin 100\%, Second and thirdgeneration cephalosporin, TrimethoprimSulfamethoxazole and Gentamicin 100\%. The least resistance by $E$. coli was observed in this study against Ciprofloxacin $14.3 \%$, Gentamicin $37.5 \%$, Ofloxacin 14.3\%. Resistance by Streptococcus spp was noted against Ampicillin $100 \%$ and Ampicillin cloxacillin 100\%, cefuroxime $100 \%$, Ciprofloxacin $100 \%$, and Trimethoprim-Sulfamethoxazole $60 \%$. The new generation macrolide (clarithromycin and azithromycin) experienced no resistance to a microorganism with known activity, unlike older generation macrolide (erythromycin) that shown acquired resistance to Streptococcus and staphylococcus aureus.

The antibiotic susceptibility pattern results to Staphylococcus aureus showed levofloxacin $100 \%$, Nitrofurantoin 100\%, Amoxicillin clavulanic acid $64 \%$. The least resistance by $E$. coli was observed in this study against Ciprofloxacin 14.3\%, Gentamicin 37.5\%, Ofloxacin $14.3 \%$. Sensitivity by Streptococcus spp was observed in levofloxacin 100\%, Nitrofurantoin 100\%, Amoxicillin Clavulanic acid $66.7 \%$, Erythromycin $66.7 \%$, and Ofloxacin $66 \%$. Ciprofloxacin and levofloxacin were active against Salmonella species, Pseudomonas species, klebsiella, and Escherichia coli. Azithromycin, imipenem, and meropenem with high susceptibility to local bacterial isolates sample tested. The access group of antibiotics, according to WHO classification, showed a low susceptibility rate to local bacteria isolate compared to the watch group with a high susceptibility rate.

\section{Discussion}

Estimating bacterial profile and its antibiotic resistance pattern is critical in establishing antibiotics stewardship, one of the key interventions necessary to curb the further emergence and spread of antimicrobial resistance (AMR). WHO in 2017 announced the Access, Watch, Reserve ("AWaRe") classification of antibiotics. The categorization is a tool for antibiotic stewardship at local, national, and global levels with the aim of decreasing antimicrobial resistance.

Urinary Tract Infection (UTI) has been found to be one of the most common bacterial infections seen in medical practice, especially in developing countries. The most predominant isolate was Staphylococcus aureus, followed by coliform (28.50\%) and Escherichia coli (17.11\%). This is consistent with findings from studies conducted in Anambra and Zaria [11], which showed that these were common pathogenic organisms of UTI [12]. One of the key factors predisposing to UTI is poor personal hygiene [12].

The highest resistance by Staphylococcus aureus was noted against Ampicillin cloxacillin, Amoxicillin, Trimethoprim-Sulfamethoxazole, Amoxicillin clavulanic acid, Gentamicin, and Cefuroxime in this study. In a study conducted in Abuth, Zaria, Nigeria had reported a pattern of resistance to ampicillin $100 \%$, ceftriaxone $72.2 \%$, and ciprofloxacin 51.5\% [13]. [14] had reported in Kuwait, MSRA isolates were resistant to ciprofloxacin (42.7\%), erythromycin, and clindamycin (42.4\%), gentamicin (38.5\%), tetracycline $(2,652$; $38.3 \%)$, and trimethoprim (33.5\%). This reflected that resistance patterns differ in a different location and the need to have an antibiogram to guide empirical treatment type and pave the way for hospital antibiotics policy programs to promote rational use of antibiotics. 
Poor hygienic environments and the absence of a relevant antibiotic policy are potential reasons for high resistance to commonly used first lines antibiotics.

High antibiotic susceptibility patterns by Staphylococcus aureus to levofloxacin, Ciprofloxacin, and Ceftriaxone were reported in this study. Previously, Nwankwo had reported a susceptibility pattern of Levofloxacin $93.7 \%$ and Amoxicillin clavulanic acid 63\% in Kano, Nigeria [15]. This may suggest that Quinolones are effective in managing Staphylococcus aureus infections and should be considered for empirical treatment in the antibiogram.

The highest resistance by $E$. coli was noted against Ampicillin 100\% and Ampicillin cloxacillin 100\%, Chloramphenicol 80\%, cefuroxime $\quad 100 \%, \quad$ TrimethoprimSulfamethoxazole $\quad 83.3 \% \quad$ Amoxicillin Clavulanic acid 55.6\%. This pattern of resistance has been shown in studies by [16], and the authored reported $100 \%$ resistance to amoxicillin/ clavulanate, cotrimoxazole, and amoxicillin in Southwest, Nigeria in 2011. Okeke observed that E. coli isolates showed resistance to ciprofloxacin (92.86\%), cotrimozazole $(92.86 \%)$, and ceftriazone (78.58\%) [5]. This was a prove that antibiotics resistance to the first choice of antibiotics to treat $E$. coli infection were growing, there was a steadily increased in resistance pattern. This finding suggests the need to develop antibiotic policies to guide the implementation of a costeffective antibiotic stewardship program in the Kwara south senatorial district.

Least resistance by E. coli was observed in this study against Ciprofloxacin 14.3\%, Gentamicin $37.5 \%$, Ofloxacin $14.3 \%$, which is consistent with Okeke N.I et al. 2007, he also observed a lower susceptibility to ofloxacin (28.57\%) [5]. On the contrary, [16] found that high resistance rates to ofloxacin, gentamycin, nalidixic acid, and tetracycline were $70 \%, 92 \%$, $96 \%$, and $88 \%$, respectively. This shows that organisms developed resistance to commonly used antibiotics in different locations and time.
This suggests the need to have an antibiogram for location and periodically update.

Resistance by Klebsiella $s p$ was observed against Ampicillin 100\% and Ampicillin cloxacillin $100 \%$, Second and third-generation cephalosporin, Trimethoprim-Sulfamethoxazole and Gentamicin $100 \%$. The least resistance was observed in this study against ciprofloxacin $20 \%$ and ofloxacin $25 \%$. This is consistent with the reports from a study conducted in 2019 in the Brazil intensive care unit of the hospital, and the author observed Klebsiella pneumoniae developed resistance to beta-lactam antibiotics, cefuroxime, cefoxitin, ceftazidime, ceftriaxone, cefepime, gentamicin, and Ciprofloxacin [4].

The authored, in a study conducted in Ebonyi State University Teaching Hospital Abakaliki, Nigeria, in 2005, observed that the most sensitive antimicrobial in his study was Ciprofloxacin with (49.4\%) followed by Gentamicin with $38.8 \%$ than Ceftazidime with $36.7 \%$ [17]. The sensitive rate demonstrated by ciprofloxacin and ofloxacin in this study and consistent with another study in eastern Nigeria suggests that these two molecules should be considered for empirical treatment in the antibiogram of the hospitals in Kwara South Senatorial district of Kwara State.

The highest resistance by Streptococcus spp was noted against Ampicillin 100\% and Ampicillin cloxacillin 100\%, cefuroxime 100\%, Ciprofloxacin 100\%, and TrimethoprimSulfamethoxazole $60 \%$. Sensitivity by Streptococcus spp was observed in levofloxacin $100 \%$, Nitrofurantoin 100\%, Amoxicillin Clavulanic acid $66.7 \%$, Erythromycin $66.7 \%$, and Ofloxacin $66 \%$.

This study was at variance to the 1997 U.S. Surveillance Study published in 1999 that reported $94 \%$ of Streptococcus. pneumoniae isolates were susceptible to amoxicillin and amoxicillin-clavulanate, $69 \%$ to azithromycin and clarithromycin, $63 \%$ cefuroxime, and $52 \%$ to cefixime [18]. The reason for this variance may be due to the Lack of a surveillance system in the Kwara senatorial district because the 
National surveillance system for antimicrobial resistance started in 2018 in Nigeria with 12 sites. Lack of AMR surveillance has promoted indiscriminate and inappropriate use of an antibiotic that led to an increase in resistance to commonly used antibiotics with the direct consequence of increases cost of health care.

This study reflected that resistance was high in the access and watched a group of antibiotics classification by WHO. The empirical antibiotic use in these hospitals is still a problem. Antibiotics are prescribed unnecessarily and empirically for complaints where no antibiotic is needed or where culture and sensitivity results could safely be awaited.

The criteria for the selection of antimicrobial agents should be determined based on the most likely pathogen and its expected resistance pattern in the locality.

Hence, there is a need for periodic monitoring of bacteria isolate and their resistance/susceptibility pattern in a locality by constantly updating antibiogram to support empirical treatment, especially for the critically ill patient before cultures result is released.

\section{References}

[1] World Health Organization (WHO). (2016). WHO | Antimicrobial resistance: global report on surveillance 2014 [Internet]. Antimicrobial resistance: global report on surveillance 2014. Available from: http://www.who.int/drugresistance/d http://www.who.int/drugresistance/documents/survei llancereport/en/\%0A

http://www.who.int/antimicrobial-

resistance/publications/surveillancereport/en/\%0A.

[2] Costelloe C, Metcalfe C, Lovering A, Mant D, Hay AD. (2010). Effect of antibiotic prescribing in primary care on antimicrobial resistance in individual patients: Systematic review and meta-analysis. Vol. 340, BMJ (Online). p. 1120.

[3] Bbosa GS, Mwebaza N, Odda J, Kyegombe DB, Ntale M. (2014). Antibiotics/antibacterial drug use, their marketing and promotion during the postantibiotic golden age and their role in emergence of

\section{Conclusion}

Most bacteria isolated from health facilities in Kwara South were resistant to the access group. The resistance to watch a group of WHO antibiotics classification is building very fast. The empirical treatment should consider antibiotics with high sensitivity to local bacterial isolates. Stakeholders should support the development of antibiogram to guide prescriptions, prevent irrational use and improve the clinical outcome of patients. Additionally, there is a need for a more sophisticated study that will encapsulate the concerns highlighted above.

\section{Acknowledgements}

The author wishes to acknowledge the Heads of departments in the units in all the hospitals. I also wish to appreciate nurses and medical officers in the wards who allow us to collect data and medical laboratory scientists to collect the data on sensitivity and resistance tests.

\section{Conflict of Interest}

The Author declares that there is no conflict of interest.

bacterial resistance. Health (Irvine Calif); 06(05): 410-25.

[4] Ferreira RL, Da Silva BCM, Rezende GS, Nakamura-Silva R, Pitondo-Silva A, Campanini EB, et al. (2019). High prevalence of multidrug-resistant klebsiella pneumoniae harboring several virulence and $\beta$-lactamase encoding genes in a brazilian intensive care unit. Front Microbiol; 10 (JAN).

[5] Okeke IN, Aboderin OA, Byarugaba DK, Ojo KK, Opintan JA. (2007). Growing problem of multidrug-resistant enteric pathogens in Africa. Emerg Infect Dis.; 13(11):1640-6.

[6] Oluyege AO, Dada AC, Ojo AM, Oluwadare E. (2009). Antibiotic resistance profile of bacterial isolates from food sold on a university campus in south western Nigeria. Vol. 8, African Journal of Biotechnology. p. 5883-7.

[7] Egwuenu A, Obasanya J, Okeke I, Aboderin O, Olayinka A, Kwange D, et al. (2018). Antimicrobial 
use and resistance in Nigeria: situation analysis and recommendations, 2017. Pan African Med J Conf Proc;2.

[8] Onubogu U, Anochie I. (2014). Empiric antibiotic prescription among febrile under-five Children in the University of Port Harcourt Teaching Hospital, Rivers State, Nigeria. Vol. 41, Nigerian Journal of Paediatrics. p. 234.

[9] Das P, Horton R. (2016). Antibiotics: Achieving the balance between access and excess. Vol. 387, The Lancet. p. 102-4.

[10] Bebell LM, Muiru AN. (2014). Antibiotic use and emerging resistance: How can resource-limited countries turn the tide? Vol. 9, Global Heart. p. $347-$ 58.

[11] Al PAE. (2016). Antimicrobial Evaluation of Bacterial Isolates from Urine Specimen of Patients with Complaints of Urinary Tract Infections in Awka, Nigeria. Int J Microbiol; 9740273. Article ID 9740273, 6 pages http://dx.doi.org/10.115, (Article ID $9740273, \quad 6 \quad$ pages http://dx.doi.org/10.1155/2016/9740273).

[12] Ehinmidu JO. (2005). Antibiotics susceptibility patterns of urine bacterial isolates in Zaria, Nigeria. Trop J Pharm Res; 2(2):223-8.

[13] Udobi CE, Obajuluwa AF, Onaolapo JA. (2013).

Prevalence and antibiotic resistance pattern of methicillin-resistant staphylococcus aureus from an Orthopaedic hospital in Nigeria. Vol. 2013, BioMed Research International.

[14] Udo EE, Boswihi SS. (2017). Antibiotic Resistance Trends in Methicillin-Resistant Staphylococcus aureus Isolated in Kuwait Hospitals: 2011-2015. Med Princ Pract; 26(5):485-90.

[15] Nwankwo EO, Nasiru MS. (2011). Antibiotic sensitivity pattern of Staphylococcus aureus from clinical isolates in a tertiary health institution in Kano, Northwestern Nigeria. Pan Afr Med J.; 8:4.

[16] Okesola, A. O., \& Aroundegbe TI. (2011). Antibiotic resistance pattern of uropathogenic Escherichia coli in South West Nigeria. African J Med Med Sci.; 40(3):235-238.

[17]CN A. (2005). Antimicrobial susceptibility pattern of Klebsiella species from Ebonyi State University Teaching Hospital Abakaliki, Nigeria. Niger J Clin Pract; 8(2):90-93.

[18] Jacobs MR, Bajaksouzian S, Zilles A, Lin G, Pankuch GA, Appelbaum PC. (1999). Susceptibilities of Streptococcus pneumoniae and Haemophilus influenzae to 10 oral antimicrobial agents based on pharmacodynamic parameters: 1997 U.S. surveillance study. Antimicrob Agents Chemother; 43(8):1901-8. 